\title{
Simulation Model of a Piston Type Hydro-Pneumatic Accumulator
}

\author{
Juho Alatalo Toni Liedes Mika Pylvänäinen \\ Mechatronics and Machine Diagnostics, University of Oulu, Finland, \\ \{juho.alatalo, toni.liedes, mika.pylvanainen\}@oulu.fi
}

\begin{abstract}
Hydro-pneumatic accumulators are used to improve the features of different kinds of hydraulic systems and they are common in industry and mobile applications. In order to include functionality of accumulators to hydraulic system models, an accurate yet light simulation model of hydro-pneumatic accumulator is needed. In this paper a simulation model of a piston type hydro-pneumatic accumulator is presented. The simulation model takes into account of the behavior of friction, nitrogen gas and hydraulic fluid. The simulation model was validated by comparing the simulation results to measurement results obtained from laboratory tests, and strong correlation was found between them. The model is suitable for researchers as well as for engineers in designing work in industry.
\end{abstract}

Keywords: gas-charged accumulator, computer simulations, modelling

\section{Hydro-pneumatic Accumulator}

Storing the energy in hydraulic fluid is very tricky. That is why there is a separate component for storing and releasing energy in hydraulic systems, an accumulator. In the accumulator energy can be bound for example to the gas pressure or potential energy of the spring. This study focuses on examining the gas-operated accumulator, so called hydro-pneumatic accumulators.

Hydro-pneumatic accumulators are conventionally utilized in hydraulic systems in several ways. One of the most common way is to improve the features of the hydraulic system, for example, in order to absorb the pressure impulses in the hydraulic line. The other main method of application for the hydro-pneumatic accumulator is to secure the safe operation of the machine, and in applications of this kind the accumulator usually operates as a reserve volume flow source. One of the main advantages of the hydro-pneumatic accumulators is their capability to act as a local and distributed energy storage and volume flow source.

Hydro-pneumatic accumulators are widely used in industry and mobile applications. In industry, the hydropneumatic accumulators are common in power plants such as windmills and wave power plants. In mobile applications, hydro-pneumatic accumulators are also common in different kinds of mobile working machines like mine loaders, harvesters and load-haul-dump machines. Hydropneumatic suspension is a widespread solution in a variety of mobile applications: cars, trucks, agricultural vehicles and military vehicles.

Three main types of accumulators exist with different kinds of properties: a diaphragm accumulator, a bladder accumulator and a hydro-pneumatic piston accumulator. Bladder and diaphragm accumulators are very much alike, but the piston type accumulator has some pros and cons compared to the two mentioned above. The main disadvantages in a piston type accumulator compared to the bladder and diaphragm type accumulators are its insensitivity to small and rapid pressure fluctuations and the greater weight of the accumulator, which may be essential in mobile machines, but not necessary in an industrial environment. The main advances in a piston type accumulator compared to the bladder and diaphragm type accumulators are its ability to handle a wider range of pressure changes and its ability to be entirely depleted from hydraulic fluid. In addition, a piston type accumulator is less sensitive to temperature changes and its installation orientation is not predefined. Also, using the sensors is easier in piston type accumulators, which means that the state of the accumulator is easier to monitor (Palomäki, 2012). In most applications all of the accumulator types mentioned above can be used, but in research and development the piston type is preferred because of its wider ranges of temperature and pressure, its modifiability, durability and the ability to be easily monitored by sensors. The structure of a piston type hydro-pneumatic accumulator is shown in in Figure 1.

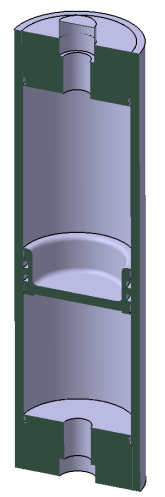

Figure 1. Cross-section of a piston type hydro-pneumatic accumulator. 


\subsection{Recent Research}

The focus of recent studies in this field is on energy consumption and energy storing, and the potential of the hydro-pneumatic accumulator in this research areas has been realized. Even though the hydro-pneumatic accumulator is an old invention, the research and development of piston type accumulators is constantly ongoing and some popular development areas can be introduced: the improvement of piston type accumulator energy storing capacity (Tavares, 2011) and the improvement of a piston type accumulator's efficiency by utilizing heat regeneration (Stroganov and Sheshin, 2011). Both these areas are closely linked with research into energy recovering systems (Achten, 2008; Ancai and Jihai, 2009; Lin et al., 2010; Zhang et al., 2010), which in particular is a contemporary research area in the field of mobile working machines.

Minav et al. (2012) have studied means of improving the energy efficiency of a mobile working machine. In their conclusion the storing of the hydraulic energy in piston type accumulators was seen as a waste of energy in a given type of energy storing method. Instead, Minav et al. proposed that energy should be stored in electric form. Their research shows the need for additional research into hydraulic systems and hydraulic energy storing methods. Instead of abandoning the idea of using hydro-pneumatic accumulators as energy storages in a mobile working machine, it would be economically reasonable to redesign and improve the old accumulator design and inefficient energy storing methods. This is due the fact that hydraulics are still widely favored in industry and especially mobile working machines due to its good power-to-weight-ratio and the ease of maintenance.

\subsection{Purpose of This Study}

Despite the simplicity of the piston type hydro-pneumatic accumulator's structure, it is fairly complex to model, because from the modelling point of view it is a multidomain system. This means that it has several different parts: mechanics, thermodynamics, pneumatics and hydraulics.

This study presents an advanced but yet feasible simulation model of a piston type hydro-pneumatic accumulator. The aim of the simulation model is to be an easy-to-use but yet accurate tool for researchers or engineers and designers in industry, for example, providing information on how an accumulator works in larger hydraulic systems, such as energy storing or suspension systems.

\section{Logical Structure of Simulation Model}

In this section the relevant physical phenomena of a piston type hydro-pneumatic accumulator are examined and the conceptual model is created on which the simulation model is based. The conceptual model is used to ensure that the logical structure of the model is correct and that all the essential elements that describe the behavior of the real hydro-pneumatic accumulator are included in the simulation model. When all the interactions between these elements are properly described, the structure of the simulation model is verified allowing the simulation model to achieve the desired accuracy.

\subsection{Physics of Piston Type Hydro-Pneumatic Accumulator}

In a piston type hydro-pneumatic accumulator, part of the energy adhered to the hydraulic fluid is converted to the energy adhered to the nitrogen gas with the help of the motion of the piston.

\subsubsection{Nitrogen gas}

In the regular duty cycle of the hydro-pneumatic accumulator, the volume $V_{\text {gas }}$, pressure $p_{\text {gas }}$ and temperature $T_{\text {gas }}$ of the nitrogen gas change and their values are dependent on each other. The volume of the gas, naturally, follows the position of the piston and volume and temperature increase and decrease in relation to the position of the piston. The nitrogen gas interacts with its environment not only via piston displacement, but also by the transition of heat energy through the piston wall. The heat exchange between the environment and the gas depend on the temperatures of the gas and the environment. In this case the environment of the gas is considered to be the interior of the cylinder wall. Also the amount of nitrogen gas usually decreases slowly over the time, due to the leakage past the piston or through the gas valve.

\subsubsection{Mechanical Structure}

There are two main mechanical structures in piston type hydro-pneumatic accumulators: the piston and cylinder wall. The piston is the only moving part of the mechanical structure and the kinetic energy is bound to the piston when it is moving. There is friction between the seal of the piston and the cylinder wall, which should be noted especially when the piston is starting to move from the rest position. The friction also heats up the piston and cylinder wall. The seal of the piston is a flexible element and together with piston inertia and static friction it causes the piston type accumulator to be insensitive to the small and high frequency fluctuations of pressure. The cylinder wall mainly acts as a thermal energy reservoir, which interacts with nitrogen gas and the ambience of the accumulator. All the mechanical parts are subject to constant changes in pressure and temperature, which affects the dimensions of these mechanical parts.

\subsubsection{Hydraulic Fluid}

The pressure, volume and the temperature of the hydraulic fluid also change during the regular duty cycle of the accumulator, but compared to nitrogen gas the changes in volume and temperature are much less significant. If the hydraulic fluid contains a lot of air bubbles, changes of volume occur at the lower pressure levels. There is also 




Figure 2. Conceptual model of a hydro-pneumatic accumulator.

one major difference between the nitrogen gas and the system that the hydraulic fluid forms: the amount of hydraulic fluid in a hydro-pneumatic accumulator varies dramatically during the regular duty cycle of the hydro-pneumatic accumulator due to the input and output flow. When flowing, the hydraulic fluid has some kinetic energy adhered to itself and also some dissipation occurs.

\subsection{Conceptual Model}

In this study the accumulator is considered to be a system that consists of several subsystems. These subsystems represent the essential elements needed to create the model. Figure 2 shows the subsystems that form the whole system of an accumulator. The subsystems are nitrogen gas, piston and hydraulic fluid. Everything outside of these subsystems is assumed to be ambient. It is important to note that some assumptions are made in order to simplify the model. It is good to keep in mind that we want the model to be feasible but yet accurate enough, so some assumptions and idealizations have to be made. One main simplification is that cylinder walls are not part of this model. The walls of the cylinder store thermal energy and play quite a significant role in the accumulator. The role of the cylinder wall can be taken into account when choosing the mathematical representation of the nitrogen gas, which will be discussed in the next section. All the material in each subsystem is predicted to be homogenous, which means that air bubbles in the hydraulic fluid are not part of the model. Changes in the dimensions of the cylinder wall and piston are not taken into account. Also the subsystem of gas is assumed to be a closed system, which means that the amount of substance in this subsystem is constant. The kinetic energy adhered to the motion of the hydraulic fluid is considered negligible.

\section{Mathematical Model}

This simulation model was made using MATLAB/Simulink, which is advisable for modelling a multi-domain system of this kind. The approach of this simulation model was chosen to be a lumped model, which at best keeps the simulation model light. In this section we will go through the equations used in this model.

\subsection{Equations for Nitrogen Gas}

In this simulation model the nitrogen gas was described with a real gas model, which has been used in several investigations. Heat transfer in a hydro-pneumatic accumulator has been studied by Els and Grobbelaar (1999), Pourmovahed and Otis (1990) and Giliomee (2003). The advantages of this real gas model compared to the commonly used ideal gas model in a piston type hydro-pneumatic accumulator have been investigated by Puddu and Paderi (2013) and Kroneld (2018) used real gas model in his research.

In this real gas model, the pressure, volume and the temperature of the gas are described using three equations. (1) describes the heat transfer between the gas and the environment,

$$
\begin{aligned}
& \dot{T}_{\mathrm{gas}}=\frac{\left(T_{\mathrm{amb}}-T_{\mathrm{gas}}\right)}{\tau_{\mathrm{therm}}}-\frac{\dot{v}}{C_{v}}\left[\frac{R T_{\mathrm{gas}}}{v}\left(1+\frac{b}{v^{2}}\right)\right. \\
& \left.+\frac{1}{v^{2}}\left(B_{0} R T_{\mathrm{gas}}+\frac{2 C_{0}}{T_{\mathrm{gas}}^{2}}\right)-\frac{2 c}{v^{3} T_{\mathrm{gas}}^{2}}\left(1+\frac{\gamma}{v^{2}}\right) e^{-\frac{\gamma}{v^{2}}}\right],
\end{aligned}
$$

where $\dot{T}_{\text {gas }}$ is change in gas temperature, $T_{\text {amb }}$ is ambient temperature, $T_{\text {gas }}$ is gas temperature, $\tau_{\text {therm }}$ is thermal time constant, $\dot{v}$ is change in specific volume of the gas, $C_{V}$ is constant volume-specific heat capacity of gas, $v$ is the specific volume of the gas and parameters $R, b, B_{0}, C_{0}$, $c$, and $\gamma$ are specific constants for nitrogen gas and their values can be found in (Giliomee, 2003).

(2) is the Benedict-Webb-Rubin -equation of state (Cooper and Goldfrank, 1967),

$$
\begin{aligned}
p_{\mathrm{gas}} & =\frac{R T_{\mathrm{gas}}}{v}+\left(\frac{B_{0} R T_{\mathrm{gas}}-A_{0}-\frac{C_{0}}{T_{\mathrm{gas}}^{2}}}{v^{2}}\right) \\
& +\left(\frac{b R T_{\mathrm{gas}}-a}{v^{3}}\right)+\frac{a \alpha}{v^{6}}+\left[\frac{c\left(1+\frac{\gamma}{v^{2}}\right) e^{\frac{-\gamma}{v^{2}}}}{v^{3} T_{\mathrm{gas}}^{2}}\right],
\end{aligned}
$$

where $p_{\text {gas }}$ is gas pressure and parameters $A_{0}, a$ and $\alpha$ are specific constants for nitrogen gas and their values can be found in (Giliomee, 2003).

(3) is the equation for the specific heat capacity of the 
gas,

$$
\begin{array}{r}
C_{v}=R\left[\frac{N_{1}}{T_{\text {gas }}^{3}}+\frac{N_{2}}{T_{\text {gas }}^{2}}+\frac{N_{3}}{T_{\text {gas }}}+\left(1-N_{4}\right)+N_{5} T_{\text {gas }}\right. \\
\left.\quad+N_{6} T_{\text {gas }}^{2}+N_{7} T_{\text {gas }}^{3}+\frac{N_{8}\left(\frac{N_{9}}{T_{\text {gas }}}\right)^{2} e^{\frac{N_{9}}{T_{\text {gas }}}}}{\left(\frac{N_{9}}{e^{T_{\text {gas }}}-1}\right)^{2}}\right],
\end{array}
$$

where parameters $N_{1}-N_{9}$ are specific constants for nitrogen gas and their values can be found in (Giliomee, 2003).

As mentioned before, the cylinder wall is not included in this model. The lack of this kind thermal energy storage can be taken into account with the thermal time constant, which must be determined by measuring. This restricts the range of use of the model to situations where the system has been settled to the thermal balance e.g. for sinusoidal use. Also the ambient temperature must be measured. The mass of the nitrogen gas must also be measured in order to calculate the specific volume of the gas.

The way how the state variables of the real gas model are connected and how they depend on the other state variables of the simulation model can be seen from Figure 3 .

\subsection{Equations for Piston}

The piston in this simulation model is constantly subjected to three different forces: the force caused by the hydraulic fluid pressure $F_{\text {hyd }}$, the force caused by the gas pressure $F_{\text {gas }}$ and friction force $F_{\mu}$. This means that the total force on piston $F_{\text {piston }}$ can be written as:

$$
\begin{aligned}
& F_{\text {piston }}=F_{\text {hyd }}- F_{\text {gas }} \pm \\
&=\Delta p_{\text {piston }} A_{\text {piston }} \pm F_{\mu} \\
& \quad=\left(p_{\text {hyd }}-p_{\text {gas }}\right) A_{\text {piston }} \pm F_{\mu},
\end{aligned}
$$

where $\Delta p_{\text {piston }}$ is pressure differentiation over the piston, $A_{\text {piston }}$ is the area of the piston, $p_{\text {hyd }}$ is pressure of the hydraulic fluid and $p_{\text {gas }}$ is pressure of the gas.

From Newton's second law we get:

$$
F_{\text {piston }}=m_{\text {piston }} \ddot{x}_{\text {piston }},
$$

where $m_{\text {piston }}$ is the mass of piston and $\ddot{x}_{\text {piston }}$ is the acceleration of piston.

The mass of the piston is easy to measure and no other parameters are needed to determine the piston.

The way the state of the piston depends on the other state variables of the simulation model of the accumulator can be seen from Figure 3 .

\subsection{Equations for Friction}

The friction in this simulation model has been described with the friction model introduced by Olsson (1996). The friction model for the pre-sliding displacement is described with the help of bristles. After the bristles have deflected a certain amount, the surfaces start to slide with respect to each other. Friction plays important role in a piston type accumulator especially when the piston is changing direction or is at rest, and without such a complex friction model the accuracy of the simulation model would not be satisfying.

The friction force $F_{\mu}$ is written as:

$$
F_{\mu}=\sigma_{0} z+\sigma_{1}\left(v_{\text {frict }}\right) \dot{z}+F_{\mathrm{v}} v_{\text {frict }},
$$

where $\sigma_{0}$ is the stiffness of the bristle, $z$ is the deflection of the bristle, $\sigma_{1}\left(v_{\text {frict }}\right)$ is velocity dependent damping coefficient, $\dot{z}$ is the rate of change of deflection of the bristle, $F_{\mathrm{v}}$ is viscous friction coefficient and $v_{\text {frict }}$ is relative velocity between surfaces.

The velocity dependent damping coefficient can be written as:

$$
\sigma_{1}\left(v_{\text {frict }}\right)=\sigma_{1} e^{-\left(\frac{v_{\text {frict }}}{v_{\mathrm{d}}}\right)^{2}},
$$

where $\sigma_{1}$ is damping coefficient and $v_{\mathrm{d}}$ is the velocity when the damping affects.

The damping coefficient $\sigma_{1}$ can be chosen by using the equation:

$$
0.5 \ldots 2 \sqrt{\sigma_{0} m_{\text {piston }}} .
$$

The rate of change of deflection of the bristle can be written as:

$$
\dot{z}=v_{\text {frict }}-\frac{\left|v_{\text {frict }}\right|}{g\left(v_{\text {frict }}\right)} z
$$

where the equation $g\left(v_{\text {frict }}\right)$ can be written as:

$$
g\left(v_{\text {frict }}\right)=\frac{1}{\sigma_{0}}\left[F_{\mathrm{C}}+\left(F_{\mathrm{S}}-F_{\mathrm{C}}\right) e^{-\left(\frac{v_{\text {frict }}}{v_{\mathrm{S}}}\right)^{2}}\right],
$$

where $F_{\mathrm{C}}$ is Coulomb friction, $F_{\mathrm{S}}$ is static friction and $v_{\mathrm{S}}$ is Stribeck velocity.

The parameters in the friction model are strongly dependent on the materials used and they must be figured out by measuring in order to make the friction model work. These measured parameters are $F_{\mathrm{C}}, F_{\mathrm{S}}, F_{\mathrm{v}}, \sigma_{0}, \sigma_{1}, v_{\mathrm{d}}$ and $v_{\mathrm{S}}$. The way the state variables of the friction model are connected to each other and how they depend on the other state variables of the simulation model of the accumulator can be seen from Figure 3.

\subsection{Equations for Hydraulic Fluid}

To figure out the pressure of the hydraulic fluid, the following equation were used (Merritt, 1967):

$$
\dot{p}_{\text {hyd }}=\frac{B_{\text {hyd }}}{V_{\text {hyd }}}\left(\sum q-\dot{V}_{\text {hyd }}\right),
$$

where $\dot{p}_{\text {hyd }}$ is the rate of change of the pressure of the hydraulic fluid, $B_{\text {hyd }}$ is the bulk modulus of the hydraulic fluid, $q$ is flow and $\dot{V}_{\text {hyd }}$ is the rate of change of the volume of the hydraulic fluid.

The pressure of the hydraulic fluid can be solved by integrating (11). The bulk modulus must be defined before this model can be used as a part of the simulation model 


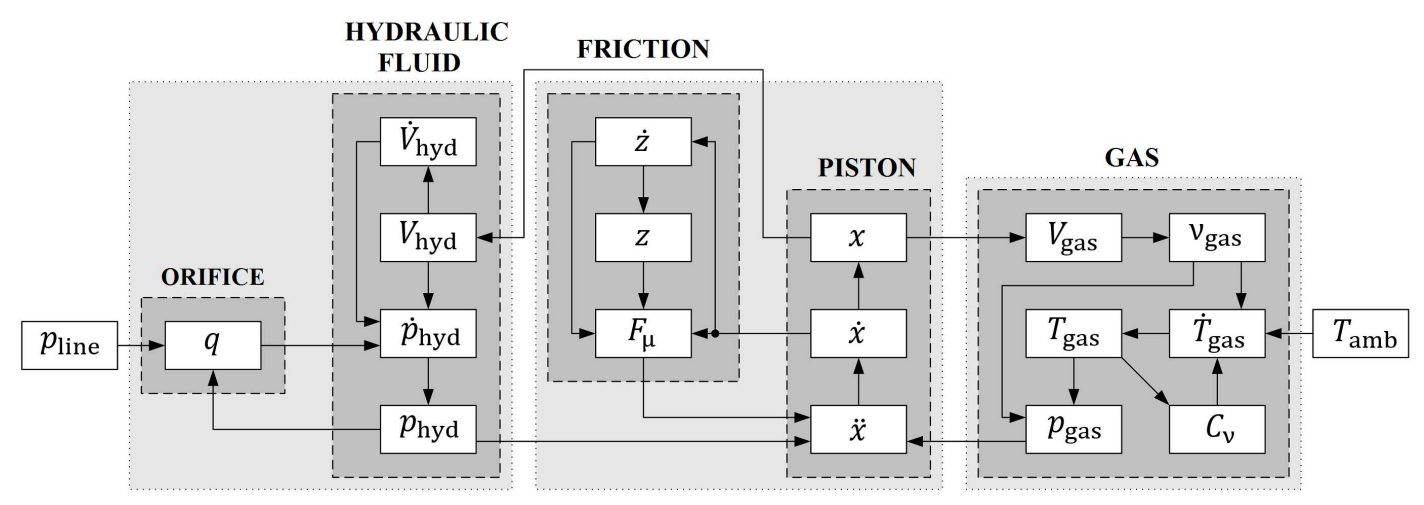

Figure 3. State variables and their connections in the simulation model.

and it is assumed to be constant. The way the state variables of the hydraulic fluid model are connected to each other and how they depend on the other state variables of the simulation model of the accumulator can be seen from Figure 3.

\subsection{Equations for Orifice}

If it is desired to use the pressure of the hydraulic line as an input for the whole simulation model, the input information is needed in order to convert to the quantity of the flow $q$ before we can utilize it in a hydraulic fluid model. For this purpose the orifice model is placed between input pressure information and hydraulic fluid model. Also this model can be used to describe the dissipation that occurs when hydraulic fluid is flowing.

The orifice model used was introduced by Åman (2011) and it is a two regional orifice model that describes the laminar flow more accurately than the commonly used turbulent flow model. The difference between these two models becomes notable especially at zero flow and when the flow is changing direction. The model describes the flow with a piecewise defined equation:

$$
q=\left\{\begin{array}{ll}
a_{1} \Delta p_{\text {orif }}+a_{2} \Delta p_{\text {orif }}^{2}+a_{3} \Delta p_{\text {orif }}^{3} & \text { if }\left|\Delta p_{\text {orif }}\right| \\
K \sqrt{\left|\Delta p_{\text {orif }}\right|} \operatorname{sign}\left(\Delta p_{\text {orif }}\right) & \text { if }\left|\Delta p_{\text {orif }}\right| \\
& \geq\left|\Delta p_{\text {trans }}\right|
\end{array},\right.
$$

where $\Delta p_{\text {orif }}$ is the pressure difference over the orifice.

When the density of the hydraulic fluid is assumed to be constant, the parameter $K$ can be written as:

$$
K=C_{\mathrm{q}} A_{\text {orif }} \sqrt{\frac{2}{\rho_{\mathrm{hyd}}}},
$$

where $C_{\mathrm{q}}$ is discharge coefficient, $A_{\text {orif }}$ is the area of the orifice and $\rho_{\text {hyd }}$ is the density of the hydraulic fluid.

According to Åman, the pressure limit $\Delta p_{\text {trans }}$ can be defined as:

$$
\Delta p_{\text {trans }}=\frac{R e^{2} v^{2} \pi \sqrt{\rho_{\text {hyd }}}}{\sqrt{32} C_{\mathrm{q}} K},
$$

where $R e$ is the Reynolds number and $v$ is kinematic viscosity of the hydraulic fluid.

The parameters $a_{1}, a_{2}$ and $a_{3}$ can be expressed as matrix A:

$$
\begin{aligned}
\mathbf{A} & =\left[\begin{array}{lll}
a_{1} & a_{2} & a_{3}
\end{array}\right] \\
& =\left[\begin{array}{lll}
\frac{5 K}{4 \sqrt{\Delta p_{\text {trans }}}} & 0 & -\frac{K}{4 \Delta p_{\text {trans }}^{\frac{5}{2}}}
\end{array}\right] .
\end{aligned}
$$

The parameters $C_{\mathrm{q}}, R e, \rho_{\text {hyd }}$ and $v$ must be defined before this model can be used as part of the simulation model. Also the parameter $A_{\text {orif }}$ must be adjusted so that the pressure drop over the orifice corresponds to the measured values. The way how the variable of the orifice model depends on the other variables of the simulation model of the accumulator is shown in Figure 3.

All the variables presented in Figure 3 can be solved either by means of the given equations or by integrating them. The inputs for the whole system are the pressure of the hydraulic line and environment temperature. If necessary, the flow can be used as an input too, but then the pressure of the hydraulic line and the orifice model must be ignored.

\section{Modelling in MATLAB/Simulink}

The layout of the simulation model built in MATLAB/Simulink follows the structure represented in Figure 3 . In order to keep the layout of the simulation model lucid, the subsystems were used in Simulink. The named blocks in Figure 3 (Orifice, Hydraulic fluid, Friction, Piston and Gas) represent the contents of these subsystems. As an example of the method how the equations in the orifice subsystem are used in Simulink is shown in Figure 4. As can be seen from Figure 4 the inputs for the subsystem are the pressure of the hydraulic line, $p_{\text {line, }}$, and the pressure of the hydraulic fluid, $p_{\text {hyd }}$. The output of this subsystem is the flow $q$. The parameters needed in the subsystem are read from a separate file, and the new set of parameters are extremely easy to feed to the model by just modifying the file where the parameters are read from. This makes the use of this simulation model flexible. The rest of the Simulink model is built by using the same kind of approach for every subsystems as described above. 


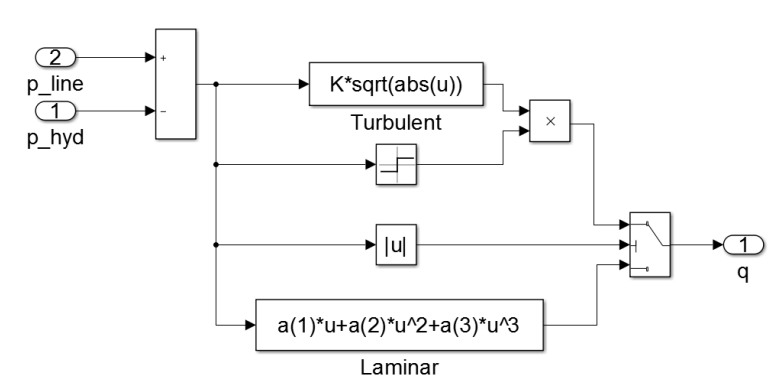

Figure 4. The orifice subsystem in Simulink.

\section{Calibration and Validation of the Simulation Model}

The equations used in the simulation model were introduced in the previous section. These equations contained unknown parameters, excluding some parameters in the real gas model, which had to be defined. In order to define these parameters for this application, a testing setup was built and measurements were performed. The goal is to determine these parameters so accurately that our accumulator model is well calibrated and its performance can be validated by comparing the simulation results to the result obtained from the measurements for the real piston type hydro-pneumatic accumulators. The parameters were determined for five different piston type hydro-pneumatic accumulators, with piston diameters $60 \mathrm{~mm}, 80 \mathrm{~mm}, 100$ $\mathrm{mm}, 125 \mathrm{~mm}$ and $140 \mathrm{~mm}$ and a nominal volume of 2 liters.

\subsection{Testing Setup}

The testing setup was built in the testing laboratory of the department of mechanical engineering at the University of Oulu. The schematic picture of the testing setup can be seen in Figure 5. The testing setup is a closed hydraulic system, which consist of a hydraulic cylinder that is directly connected to the piston type hydro-pneumatic accumulator. There is also a parallel diaphragm hydropneumatic accumulator that is only used in certain measurement runs. The piston of the hydraulic cylinder is driven by a hydraulic servo.

The pressure of the hydraulic fluid was measured directly from the bottom of the hydraulic cylinder and the piston type hydro-pneumatic accumulator and the pressure of the nitrogen gas was measured from the gas side of the piston type hydro-pneumatic accumulator. The pressures were measured using IFM PT9541 sensors. The position of the piston of the hydro-pneumatic accumulator was measured with a non-contact sensor in order not to disturb the performance of the accumulator with additional friction. The position of the piston was measured with a Temposonics MH Series sensor. Also the position of the servo cylinder and the outside temperature were measured. The servo position sensor was HBM 1-WA/500MM-L and the temperature was measured with an ordinary K-type ther-

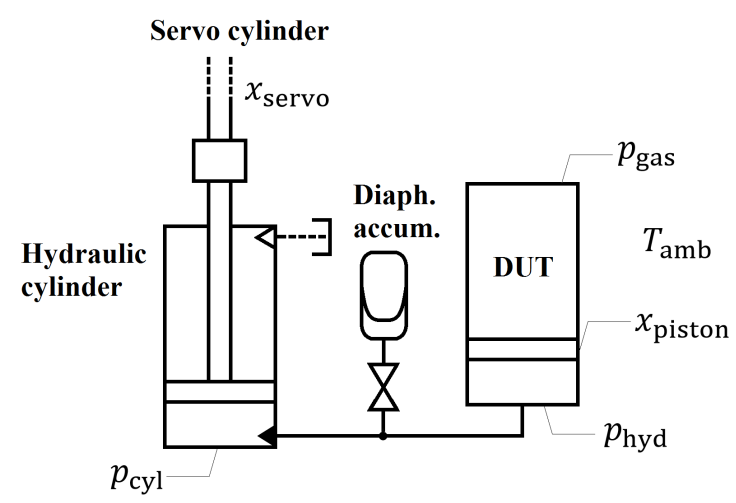

Figure 5. Schematic figure of the testing setup.

mocouple. The wall of the $140 \mathrm{~mm}$ diameter accumulator was so thick that the Temposonics sensor was unable to measure the position of the piston. Instead, the position of the piston of the $140 \mathrm{~mm}$ diameter accumulator was calculated from the position of the servo cylinder and by estimating the compression of the hydraulic fluid. The data acquisition was performed using NI cDAQ-9174, with NI 9215 and NI 9211 modules and with $250 \mathrm{~Hz}$ sampling rate. The variables at Figure 5 represent the measured quantities.

\subsection{Laboratory Tests}

Three different kinds of calibration runs were performed with the testing setup in order to determine the parameters named in Section 3.

The parameters $F_{\mathrm{C}}, F_{\mathrm{S}}, F_{\mathrm{v}}, v_{\mathrm{d}}$ and $v_{\mathrm{S}}$ for the friction model were determined with the sinusoidal motion input excitation of the servo cylinder. The Stribeck curve could be created from the information on pressure differential over the hydro-pneumatic piston and the position information of the piston. The values for the parameters could be derived from the Stribeck curve.

The parameters $\sigma_{0}$ and $\sigma_{1}$ for the friction model were determined with the help of a diaphragm hydro-pneumatic accumulator. The pressure in the hydraulic system was raised slowly and the pressure and the maximum presliding deflection of the piston seals were measured. From this information the desired parameters could be derived.

To determine the thermal time constant $\tau_{\text {therm }}$ of the piston type hydro-pneumatic accumulator, step-type excitation was introduced to the accumulator. The pressure and the volume of the nitrogen gas was measured and the value of the $\tau_{\text {therm }}$ in the simulation model was iterated in so that the pressure of the simulation model behaved accurately enough.

\subsection{Validation}

The simulation models were validated by comparing their results to the measured values. Measured and simulated results are presented Figure 6 - Figure 9 and as can be seen, the simulation results correspond really well to the 
measured data. Some of the curves appear to be one on the other, but on the $p V$ chart some difference can be seen. Note that in Figure 8 the position of piston is estimated.

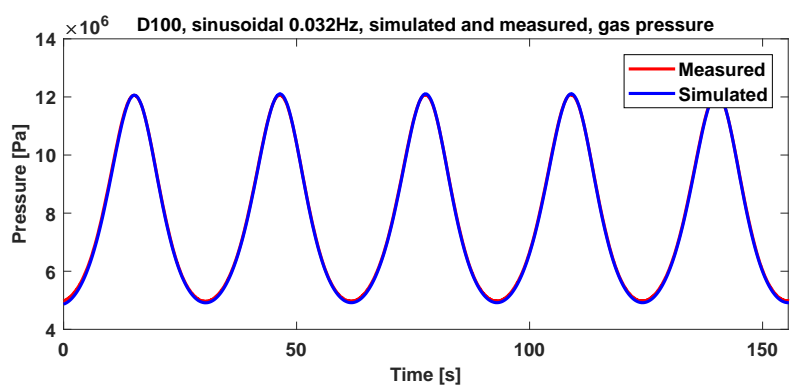

Figure 6. The gas pressures of the $100 \mathrm{~mm}$ diameter accumulator when the input for the accumulator is the sinusoidal 0.032 Hz flow.

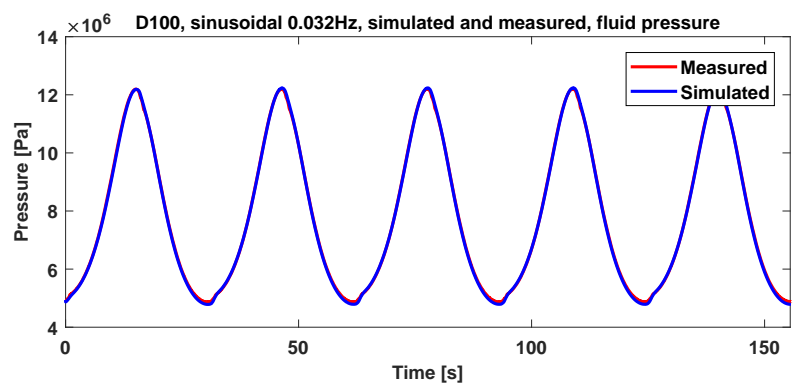

Figure 7. The hydraulic fluid pressures of the $100 \mathrm{~mm}$ diameter accumulator when the input for the accumulator is the sinusoidal $0.032 \mathrm{~Hz}$ flow

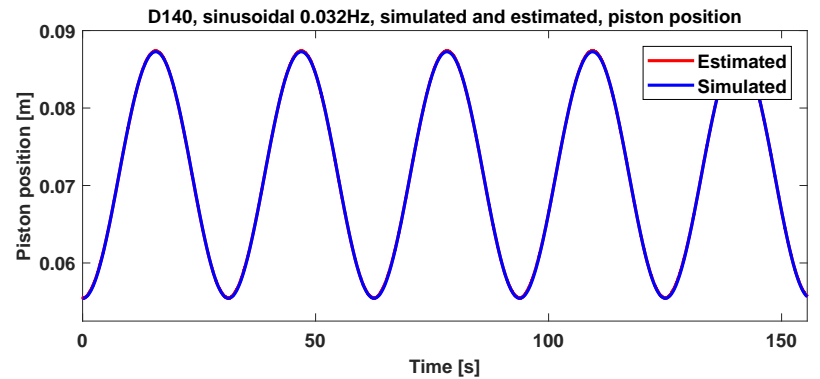

Figure 8. The piston position of the $140 \mathrm{~mm}$ diameter accumulator when the input for the accumulator is the sinusoidal 0.032 $\mathrm{Hz}$ flow.

\section{Conclusions}

The simulation model of the piston type hydro-pneumatic accumulator met the demand for an easy-to-use but yet accurate simulation model and it can be used either in research or in mechanical design work. The results of the simulation model were very accurate and there was strong correlation between the simulation and the measured data. The model itself appeared to be fairly easy to simulate. The simulation model is capable of simulating the perfor-

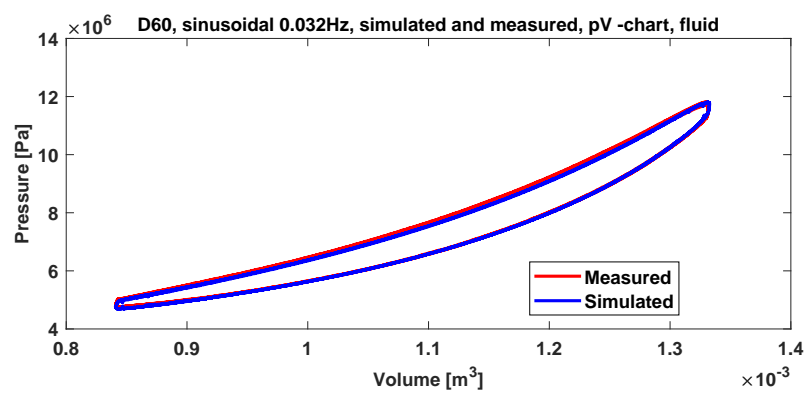

Figure 9. The $p V$ chart of the $60 \mathrm{~mm}$ diameter accumulator when the input for the accumulator is the sinusoidal $0.032 \mathrm{~Hz}$ flow.

mance of the piston type hydro-pneumatic accumulator in situations where the system is in thermal balance.

Real-time model-based condition monitoring can be regarded as an important method in future and these kinds of simulation models of different components play an important role in it. This simulation model provides a good foundation when heading towards this new practice.

There is need for further development in modelling the cylinder walls, which probably also allows the possibility to simulate situations where the accumulator is on the transition between thermal balance situations, thus extending the range of use of the simulation model.

\section{References}

P. A. J. Achten. A serial hydraulic hybrid drive train for off-road vehicles. In Proceeding $s$ of the National Conference on Fluid Power, volume 51, pages 515 - 521, 2008.

Y. Ancai and J. Jihai. Research on the regenerative braking control strategy for secondary regulation hydrostatic transmission excavators. In Proceedings of the 2009 IEEE International Conference on Mechatronics and Automation, pages 4600 - 4604, 2009.

H. W. Cooper and J. C. Goldfrank. B-W-R constants and new correlations. Hydrocarbon Processing, 46(12):141 - 146, 1967.

P. S. Els and B. Grobbelaar. Heat transfer effects on hydropneumatic suspension systems. Journal of Terramechanics, 36: $197-205,1999$.

C. L. Giliomee. Analysis of a Four State Switchable HydroPneumatic Spring and Damper System. PhD thesis, University of Pretoria, 2003.

P. Kroneld. Energy Managment in Active Suspension Systems. $\mathrm{PhD}$ thesis, University of Oulu, 2018. [unpublished].

T. Lin, Q. Wan, B. Hu, and W. Gong. Research on the energy regeneration systems for hybrid hydraulic excavators. $A u$ tomation in Construction, 19:1016 - 1026, 2010.

H. E. Merritt. Hydraulic control systems. New York : Wiley, 1967. ISBN-13:978-0471596172. 
T. A. Minav, A. Virtanen, L. Laurila, and J. Pyrhönen. Storage of energy recovered from an industrial forklift. Automation in Construction, 22:506 - 515, 2012.

H. Olsson. Control Systems with Friction. PhD thesis, Lund Institute of Technology, 1996.

H. Palomäki. Paineakkujen testilaitteiston suunnittelu. Master's thesis, Tampere University of Technology, 2012. [in Finnish].

A. Pourmovahed and D. R. Otis. An experimental thermal timeconstant correlation for hydraulic accumulators. Journal of Dynamic Systems, Measurement, and Control, 112(1):116 121, 1990.

Pi. Puddu and M. Paderi. Hydro-pneumatic accumulators for vehicles kinetic energy storage: Influence of gas compressibility and thermal losses on storage capability. Energy, 57: $326-335,2013$.

R. Åman. Methods and Models for Accelerating Dynamic Simulation of Fluid Power Circuits. PhD thesis, Lappeenranta University of Technology, 2011.

A. Stroganov and L. Sheshin. Improvement of heat-regenerative hydraulic accumulators. Ventil, 17(4):322 - 332, 2011.

F. T. Tavares. Thermally Boosted Concept for Improved Energy Storage Capacity of a Hydro-Pneumatic Accumulator. PhD thesis, University of Michigan, 2011.

X. Zhang, S. Liu, Z. Huang, and L. Chen. Research on the system of boom potential recovery in hydraulic excavator. In Proceedings of 2010 International Conference on Digital Manufacturing \& Automation, volume 2, pages 3030 - 306, 2010. 